\title{
Comparative of Interpolators Applied to Depth Images
}

\author{
Beatriz Juárez Arreortúa, Hugo Jiménez Hernández, and \\ Diana Margarita Córdova Esparza \\ Center for Engineering and Industrial Development, Querétaro, Qro., México \\ \{beatriz.juareza, hugojh, diana_mce\}@gmail.com
}

\begin{abstract}
Interpolators are widely used in image processing because they allow us to estimate the unknown values of sensor measurements. In this research, we present a comparison between commonly used interpolators to evaluate how each affect the behavior of the data, it is studied the problem of interpolation as a means to infer information at a higher frequency through the mathematical description an depth image.
\end{abstract}

Keywords: Interpolation, Depth images, Frequency.

\section{Introduction}

Image processing and analysis is currently better known and used for various activities in the world of technology, the type of analysis that is performed and the techniques used are directly linked to the amount of information provided by each image, as well as what needs to be identified through this one.

This information depends in turn on the sampling frequency of the sensor being used; a digital image is constituted by a spatial sampling of a set of sensors represented by a matrix. However, when talking about sampling, the main limitation observed is the sensor acquisition frequency, which in turn is limited due to the characteristics of the phenomenon being sampled. In several occasions to compensate for the limitations above, it is common the use of interpolators, so that we can obtain more information about the phenomenon being analyzed.

Therefore, when using an interpolator it is expected that the information obtained through it will be consistent with the data originally acquired, that is, that it does not deform the nature of the information, since this can directly affect the result of the analysis performed.

Therefore, in this work, we study the problem of interpolation as a means to infer information at a higher frequency. We examined how three different types of interpolators affect the data acquired at a certain frequency. We present experiments with depth images of increased resolution and analyze how much it affects the method of interpolation used in the original image. 


\section{Theoretical Foundation}

There is evidence in the literature of different comparatives between interpolators, however these are based on criteria such as: execution time, precision, clarity of the image, among others [1], [2]. However, for purposes of this work, what is interesting is to know how much it affects or not the use of some interpolator to the original distribution of the depth image.

In this work, three commonly used interpolators are used in image analysis, which are described below.

\subsection{Linear Interpolation}

One of the most used interpolators is the linear one described in Eq. (1) due to its simplicity. It consists in fitting a line to two given points:

$$
g(x)=\frac{b-x}{b-a} f(a)+\frac{x-a}{b-a} f(b) .
$$

where $\mathrm{g}(\mathrm{x})$ denotes that this is a first-degree interpolation polynomial. To interpolate an image, the function is first applied to the $\mathrm{x}$-axis and then to the $\mathrm{y}$-axis.

The advantage of using the linear interpolator is that the implementation is simple, for this reason, the computation time is small compared to other interpolators. Another advantage of the linear interpolation is that the results are more accurate with smaller intervals between the two points. However, in the same way, if the interval is large, the result is more inaccurate. It should also be considered that if the selected points do not correspond to a straight line, the calculated values become incorrect.

\subsection{Lagrange Interpolation}

The Lagrange interpolation polynomial is a reformulation of Newton's polynomial that avoids the calculation of the divided differences, and is represented by Eq. (2) of polynomial bases of Lagrange (Eq. 3):

$$
\begin{gathered}
f(x)=\sum_{j=0}^{k} y_{i} l_{i}(x) . \\
l_{j}(x)=\prod_{i=0, i \neq j}^{n} \frac{x-x_{i}}{x_{j}-x_{i}}=\frac{x-x_{0}}{x_{j}-x_{0}} \ldots \frac{x-x_{0}}{x_{j}-x_{0}} \frac{x-x_{0}}{x_{j}-x_{0}} \ldots \frac{x-x_{0}}{x_{j}-x_{0}} .
\end{gathered}
$$

The Lagrange interpolation grows fast computationally with the increase of the interpolator degree. The polynomial degree varies according to the input points, i.e., if we remove or add points it is necessary to change the degree of the polynomial.

\subsection{Basic Splines (B-Splines)}

The purpose of this interpolator is to make the interpolation curve smoother and improving the image edges.

The cubic B-spline function is defined in Eq. (4): 


$$
f(x)=\sum_{k=-\infty}^{\infty} B_{k, n+1}(x) \cdot f\left(x_{k}\right) .
$$

The three-order B-spline function is as follows:

$$
B_{i, 3}= \begin{cases}\frac{\left(x-x_{i}\right)^{2}}{\left(x_{i+1}-x_{i}\right)\left(x_{i+2}-x\right)\left(x-x_{i+1}\right)} . & , x_{i} \leq x \leq x_{i+1} \\ \frac{\left(x-x_{i}\right)^{2}}{\left(x_{i+1}-x_{i}\right)\left(x_{i+2}-x_{i}\right)}-\frac{\left(x_{i+2}-x\right)\left(x-x_{i+1}\right)}{\left(x_{i+2}-x_{i+1}\right)\left(x_{i+3}-x_{i+1}\right)}, & , x_{i+1} \leq x \leq x_{i+2} \\ \frac{\left(x-x_{i+3}\right)^{2}}{\left(x_{i+3}-x_{i+1}\right)\left(x_{i+3}-x_{i+2}\right)} . & , x_{i+2} \leq x \leq x_{i+3}\end{cases}
$$

The B-spline interpolator has a greater mathematical complexity, because the base functions do not support an explicit expression and change when adjusting the nodes vector.

\section{Methodology}

For this work and because they are analyzing depth images it is decided to choose two objects that have the following properties.

1. Rigid object of known form, non-deformable.

2. Soft and amorphous object.

This is considered as the most special cases that can be found when acquiring an image through a ToF camera.
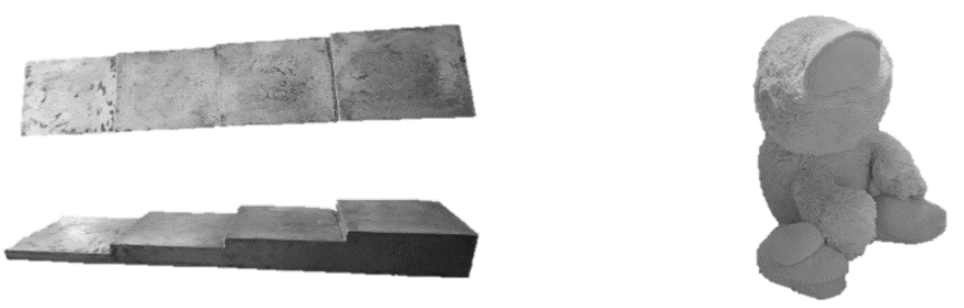

Fig. 1. Objects acquired with the depth sensor

Depth images were acquired with a ToF camera, to reduce the noise generated by the sensor, 250 depth images were acquired and we averaged to estimate the expected value of each pixel.

Were implemented and applied the three interpolators to the acquired images (Linear, Lagrange, and B-spline), increasing the acquisitions two, four, eight and sixteen times, to analyze the effect of each interpolator and each increase over the information provided in the original image.

To describe the original image, we calculated the central moments and compared with the central moments of the interpolated images. The formula of the central moment of order $\mathrm{k}$ is described in Eq. (6):

$$
\mu_{k}=E\left[(X-E[X])^{k}\right] .
$$




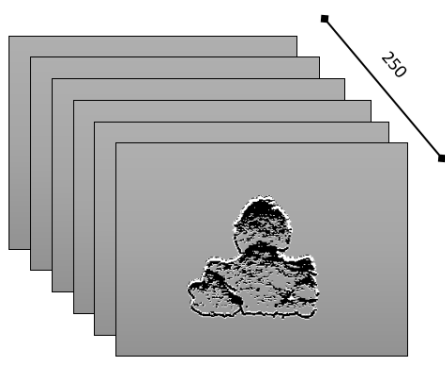

Fig. 2. Frames acquired by object

Where $\mathrm{E}$ is the expectation operator and $\mathrm{k}$ is the order of the statistical moment that is being calculated.

\section{$4 \quad$ Results}

In this section, we present the results obtained by applying the three different interpolators on the acquired original image, both qualitative and quantitative:

In the Figure 3 and 4 the interpolation applied to the original images of the plush doll and the calibration pattern respectively is shown, in both only a fragment of the image is shown as an example so that the effect of the interpolation can be better observed.

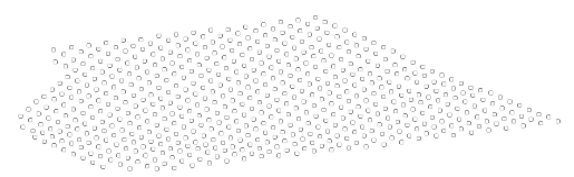

a)

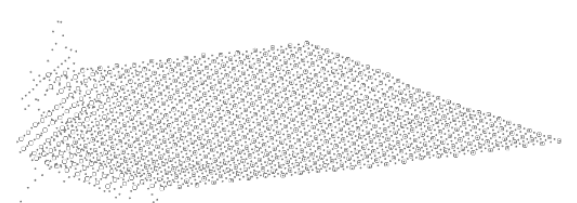

c)

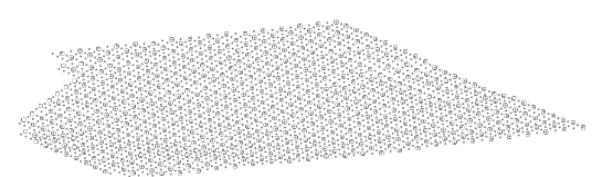

b)

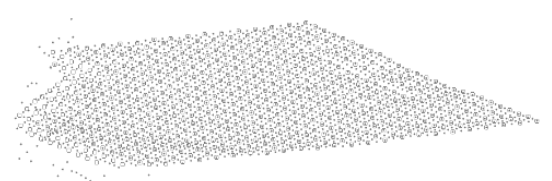

d)

Fig. 3. (a) Original image (plush doll), (b) Image with Linear Interpolator (plush doll), (c) Image with Lagrange Interpolator (plush doll), (d) Image with B-Spline Interpolator (plush doll)

In tables 1-6 we present the descriptors of the first four central moments to compare the interpolated images with the input image. Row OI represents the information obtained from the original image, and the subsequent rows represent the central mo- 
ments of the interpolated images by increasing the frequency $2,4,8$ and 16 times the original with each interpolator.

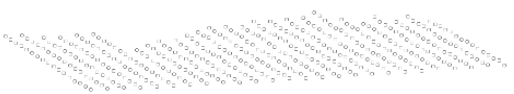

a)

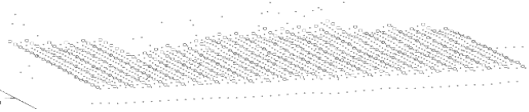

c)

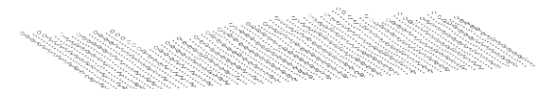

b)

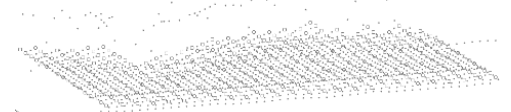

d)

Fig. 4. (a) Original image (pattern depth), (b) Image with Linear Interpolator (pattern depth), (c) Image with Lagrange Interpolator (pattern depth), (d) Image with B-Spline Interpolator (pattern depth)

Figures 5,6 and 7 depict visually how the moments of the scaled images vary more frequently with respect to the original moments of the depth image(plush doll).

Table 1. Linear interpolation (plush doll)

\begin{tabular}{|l|l|l|l|l|}
\hline & $1^{\text {st }} \mathrm{M}$ & $2^{\text {nd }} M$ & $3^{\text {rd }} \mathrm{M}$ & $4^{\text {th }} \mathrm{M}$ \\
\hline $\mathrm{OI}$ & 0.055920057 & 4567.730957 & 106484.9375 & 76746880 \\
\hline $2 \mathrm{x}$ & 0.058058724 & 4518.415039 & 111223.5469 & 75409888 \\
\hline $4 \mathrm{x}$ & 0.291386068 & 4520.250488 & 111087.3047 & 75139080 \\
\hline $8 \mathrm{x}$ & 2.15897727 & 4531.534668 & 146713.9219 & 76388360 \\
\hline $16 \mathrm{x}$ & 8.629109383 & 4570.442383 & 1114.875366 & 72706552 \\
\hline
\end{tabular}

Table 2. B-Spline interpolation (plush doll)

\begin{tabular}{|l|l|l|l|l|}
\hline & $1^{\text {st }} \mathrm{M}$ & $2^{\text {nd }} \mathrm{M}$ & $3^{\text {rd }} \mathrm{M}$ & $4^{\text {th }} \mathrm{M}$ \\
\hline $\mathrm{OI}$ & 0.055920057 & 4567.730957 & 106484.9375 & 76746880 \\
\hline $2 \mathrm{x}$ & 0.01438925 & 5114.751465 & 1607.946045 & 128895192 \\
\hline $4 \mathrm{x}$ & 0.210121885 & 5103.547852 & 28430.24414 & 129927656 \\
\hline $8 \mathrm{x}$ & 2.253632307 & 5101.760254 & 7790.346191 & 129600160 \\
\hline $16 \mathrm{x}$ & 7.980696201 & 5140.467285 & 149802.7813 & 132150752 \\
\hline
\end{tabular}

Table 3. Lagrange interpolation (plush doll)

\begin{tabular}{|l|l|l|l|l|}
\hline & $1^{\text {st }} \mathrm{M}$ & $2^{\text {nd }} \mathrm{M}$ & $3^{\text {rd }} \mathrm{M}$ & $4^{\text {th }} \mathrm{M}$ \\
\hline $\mathrm{OI}$ & 0.0559200569987 & 4567.73095703125 & 106484.937500000 & 76746880 \\
\hline $2 \mathrm{x}$ & 0.0138180907815 & 5374.58984375000 & 242783.953125000 & 261666128 \\
\hline $4 \mathrm{x}$ & 0.2379906475543 & 5420.54345703125 & 230595.296875000 & 246231024 \\
\hline $8 \mathrm{x}$ & 2.1160101890564 & 5416.71679687500 & 186943.125000000 & 241594672 \\
\hline $16 \mathrm{x}$ & 8.2407598495483 & 5457.23486328125 & 352931.562500000 & 250968080 \\
\hline
\end{tabular}




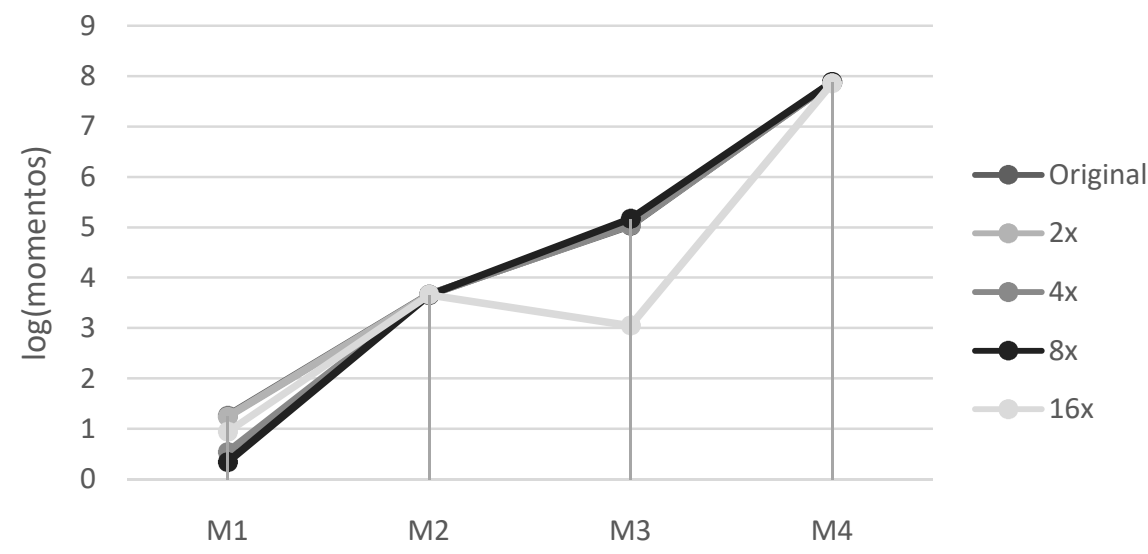

Fig. 5. Lineal interpolator

In Figure 5 it is observed that the linear interpolator for this type of images, offers good results, however, when we scale $16 x$ the original data a considerable variation is observed for the moment 3 .

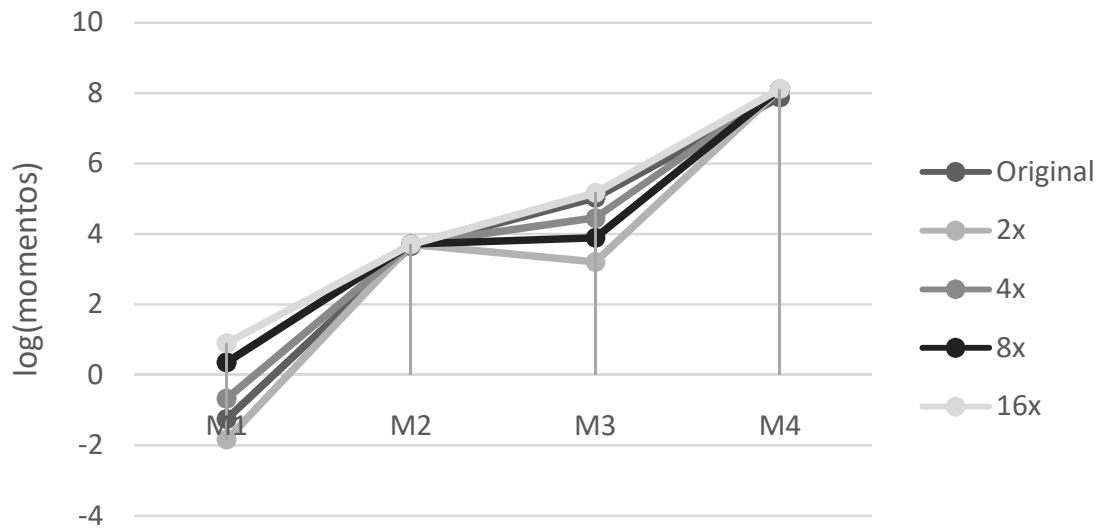

Fig. 6. B-Spline interpolator

Both the B-Spline interpolator and the Lagrange interpolator, from scaling $2 \mathrm{x}$, show a noticeable variation with respect to the original data; however, the B-Spline interpolator as seen in Figure 6 varies more than the Lagrange interpolator Figure 7 for moment 3.

Figures 5, 6 and 7 depict visually how the moments of the scaled images vary more frequently with respect to the original moments of the depth image(pattern of depth). 


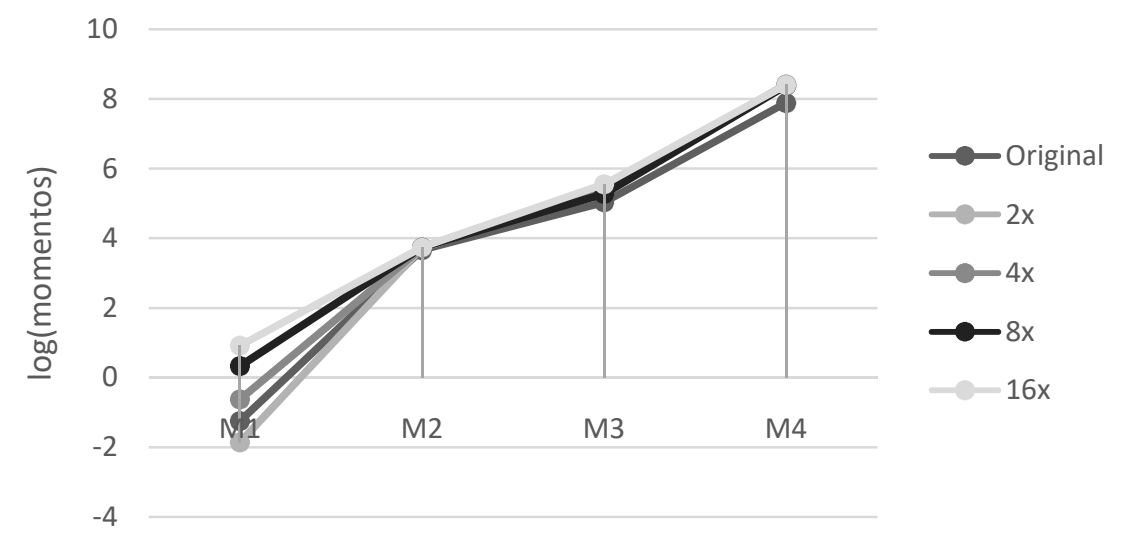

Fig. 7. c) Lagrange interpolator

Table 4. Liner interpolation (pattern of depth)

\begin{tabular}{|l|l|l|l|l|}
\hline & $1^{\text {st }} \mathrm{M}$ & $2^{\text {nd }} \mathrm{M}$ & $3^{\text {rd }} \mathrm{M}$ & $4^{\text {th }} \mathrm{M}$ \\
\hline OI & 0.0001281092700 & 60.22936633 & 569.4663696 & 11182.77637 \\
\hline $2 \mathrm{x}$ & 0.0001294422447 & 62.19714737 & 555.3723755 & 10825.80664 \\
\hline $4 \mathrm{x}$ & 0.000847409 & 63.29848484 & 524.324462890 & 10410.88477 \\
\hline $8 \mathrm{x}$ & 0.00371421 & 65.824699401 & 763.623840332 & 13556.16309 \\
\hline $16 \mathrm{x}$ & 0.047592497 & 84.282928466 & 1455.68054199 & 27358.07422 \\
\hline
\end{tabular}

Table 5. B-Spline interpolation (pattern of depth)

\begin{tabular}{|l|l|l|l|l|}
\hline & $1^{\text {st }} \mathrm{M}$ & $2^{\text {nd }} \mathrm{M}$ & $3^{\text {rd }} \mathrm{M}$ & $4^{\text {th }} \mathrm{M}$ \\
\hline OI & 0.0001281092700082 & 60.22936630249 & 569.46636962890 & 11182.7763 \\
\hline $2 \mathrm{x}$ & 0.0092535801231861 & 3264.8283691406 & 612950.50000000 & 174173216 \\
\hline $4 \mathrm{x}$ & 0.0305372662842274 & 3582.5812988281 & 626727.62500000 & 171449104 \\
\hline $8 \mathrm{x}$ & 0.204221203923225 & 3606.1777343750 & 628083.93750000 & 172152064 \\
\hline $16 \mathrm{x}$ & 3.49978852272034 & 3622.1728515625 & 588958.06250000 & 163265120 \\
\hline
\end{tabular}

Table 6. Lagrange interpolation (pattern of depth)

\begin{tabular}{|l|l|l|l|l|}
\hline & $1^{\text {st }} \mathrm{M}$ & $2^{\text {nd }} \mathrm{M}$ & $3^{\text {rd }} \mathrm{M}$ & $4^{\text {th }} \mathrm{M}$ \\
\hline OI & 0.0000000 & 0.0060229 & 0.0569466 & 1.1182776 \\
\hline $2 \mathrm{x}$ & 0.0000000 & 0.0000562 & 0.0175613 & 6.9509043 \\
\hline $4 \mathrm{x}$ & 0.0000000 & 0.0000605 & 0.0165664 & 6.1430662 \\
\hline $8 \mathrm{x}$ & 0.0000000 & 0.0000608 & 0.0164137 & 6.1218336 \\
\hline $16 \mathrm{x}$ & 0.0000000 & 0.0000609 & 0.0158865 & 5.9289088 \\
\hline
\end{tabular}


Beatriz Juárez Arreortúa, Hugo Jiménez Hernández, Diana Margarita Córdova Esparza

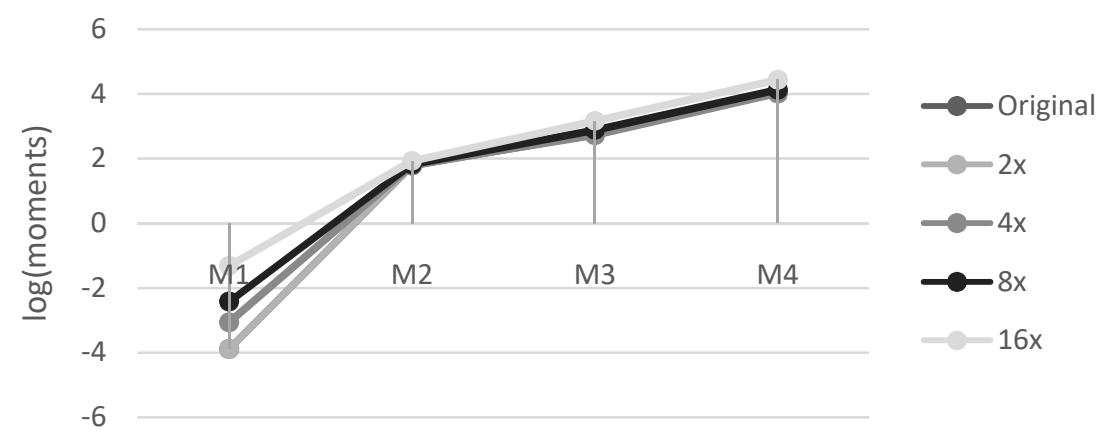

Fig. 8. Lineal interpolator

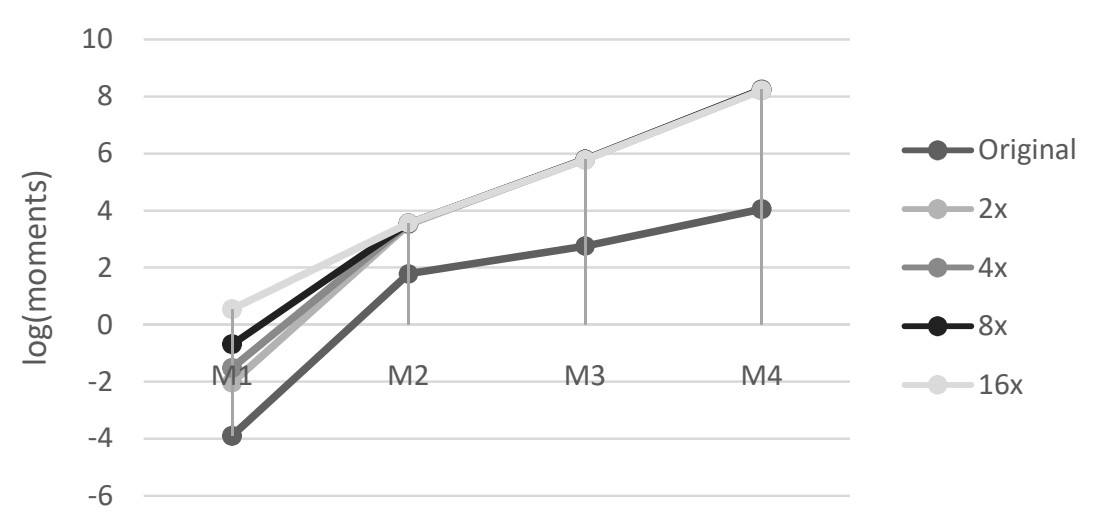

Fig. 9. B-Spline interpolator

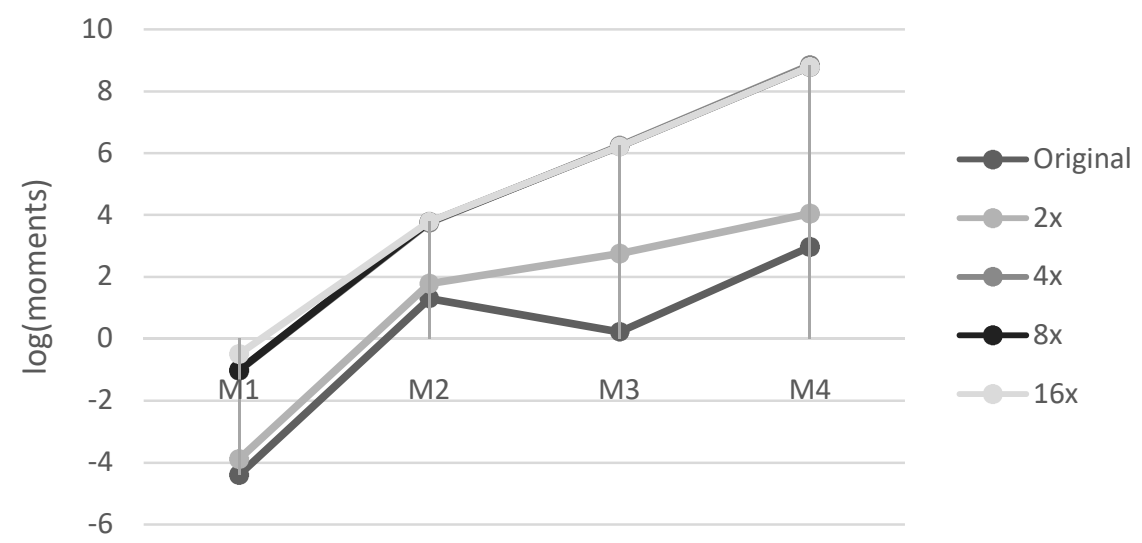

Fig. 10. Lagrange interpolator 


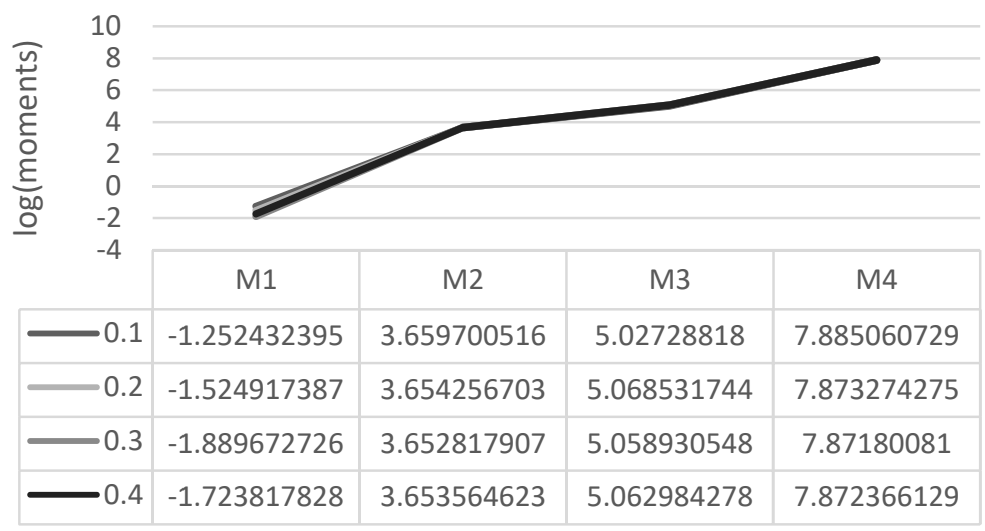

Fig. 11. Linear Interpolation

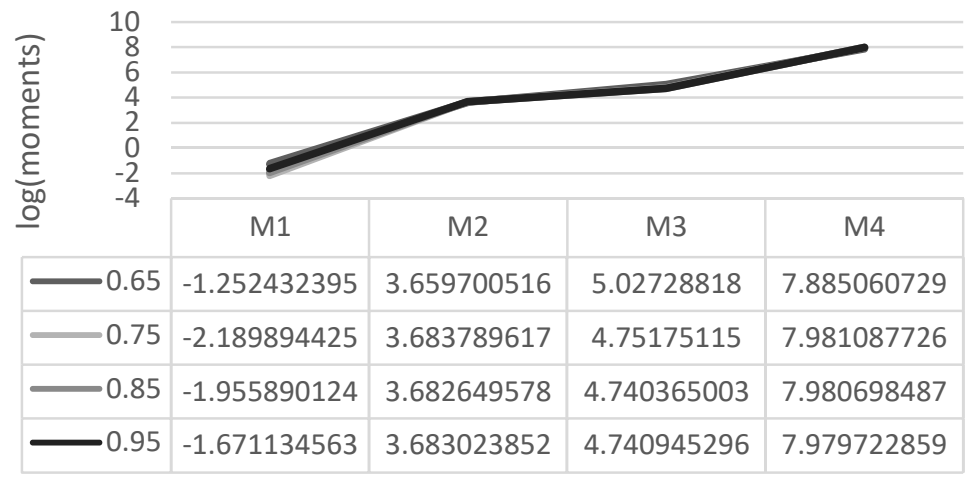

Fig. 12. B-Spline Interpolation

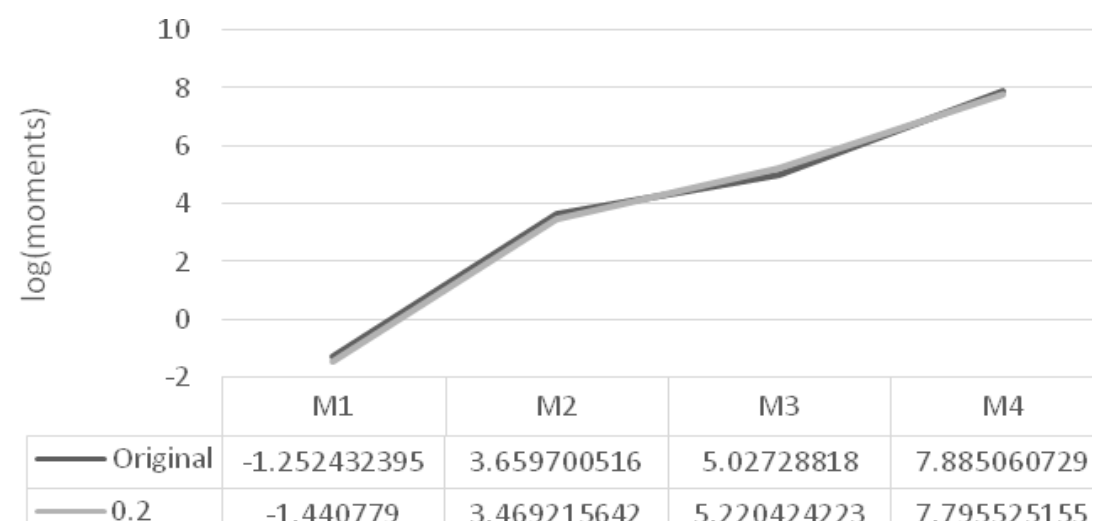

Fig. 13. Lagrange Interpolation 


\section{Conclusions}

In this project, we performed a comparison between the variation of the central moments initials of a depth image against the central moments obtained from a processed image using three different interpolation methods.

Quantitatively it can be observed that there is variation in the central moments of the original image given the selected interpolator as seen in Tables 1-6; however, it is also observed that it depends on the shape of the object being analyzed.

As can be seen in Fig. 5.6 and 7 it can be seen that the linear interpolator is the one that best fits the original central moments of the image, however, increasing it by $16 \mathrm{x}$ for the moment 3 is considerably different from the original.

As observed both the Lagrange interpolator and the B-Spline interpolator are the one that presents the greatest variation with respect to the original information as seen in Figure 6, 7, 9 and 10.

Because of this, the image is scaled with a smaller frequency to be able to visualize where it begins to move away from the original moments Figure 11, 12, 13, so that when processing images of depth from ToF sensors, this is taken into consideration.

\section{References}

1. M. K. Ng., H. Shen, E. Y. Lam, L. Zhang.: A total variation regularization based superresolution reconstruction algorithm for digital video. In: EURASIP J. Adv. Signal Process, pp. 1-16 (2007)

2. Park J., Kim H., Tai YW., Brown MS., Kweon IS.: High-quality depth map upsampling and completion for RGB-D cameras. IEEE Trans Image Process 23(12), pp. 55-72 (2014)

3. Keys R. G.: Cubic convolution interpolation for digital images. IEEE Trans. Acoust. Proceedings of Signal Processing and Communications SPC 29, 1153-1160 (1981)

4. Unser M., Aldroubi A., Eden M.: Fast B-spline transforms for continuous image representation and interpolation. IEEE Trans. Pattern Anal. Mach. Intell. 13(3), 277-285(1991)

5. Battiato S., Gallo G., Stanco F.: A locally adaptive zooming algorithm for digital images. Image Vis, 20(11), 805-812 (2002)

6. Grevera G. J., Udupa J. K.: Shape-based interpolation of multidimensional grey-level images. IEEE Trans. Med. Imag 15(6), 881-892 (1996)

7. Haralick R. M., L Watson: A facet model for image data. Comput. Graphics Image Processing 15, 113-129 (1981)

8. Chuah C. S., Leou J. J.: An adaptive image interpolation algorithm for image/video. Processing Pattern Recognition 34(12), 2383-2393 (2001)

9. Hou H. S., Andrews H. C.: Cubic splines for image interpolation and digital filtering. IEEE Trans. Acoust., Speech, and Signal Processing 26, 508-517 (1978)

10. W. Zhe, Z. Jiefu, Z. Mengchu: A Fast Autoregression Based Image Interpolation Method. In: IEEE International Conference on Networking, Sensing and Control, pp.1400-1404 (2008)

11. Rogerio, Siji Higa, Roger, Freday: Plenoptic image compression comparison between JPEG JPEG2000 and SPITH. In: Indian Conference on Computer Vision Graphics and Image Processing (ICVGIP), pp. 1-6 (2014) 
Comparative of Interpolators Applied to Depth Images

12. Sakamoto T., Kodama K., Hamamoto T.: A study on efficient compression of multi-focus images for dense Light-Field reconstruction. In: IEEE Visual Communications and Image Processing, pp. 1-6 (2012) 